\title{
Mit Information, Interaktion und Kommunikation sicher in die Freiberuflichkeit
}

Es gibt viele gute Gründe für eine Reise nach Berlin - Ende September gibt es in diesem Jahr noch einen mehr: Der Freie Verband Deutscher Zahnärzte (FVDZ) lädt zu seinem ersten „Forum Zukunft" in die deutsche Hauptstadt ein. Interaktiv und kommunikativ wird es bei dem zweitägigen Treffen am 30. September und 1. Oktober um die sichere Zukunftsplanung für junge Zahnärztinnen und Zahnärzte gehen. „Wir wollen den Teilnehmern einerseits ein prall gefülltes Informationspaket mitgeben und gleichzeitig viel Raum dafür lassen, persönliche Fragen im Austausch mit den Referenten und anderen Teilnehmern zu klären“, erläutert die stellvertretende FVDZ-Bundesvorsitzende Dr. Gudrun Kaps-Richter das neue Format.

Das Forum Zukunft startet mit einer Führung durch den Bundesrat am Nachmittag des Freitags (30. September). Mit einem Impulsreferat zum Engagement in der Kollegenschaft wird Forum-Organisatorin Kaps-Richter für den ersten Austausch der Teilnehmer vor einem gemeinsamen Abendessen sorgen. Am Samstag, 1. Oktober, geht es dann ab 9 Uhr direkt in medias res der vier Themenschwerpunkte: Rechtsanwältin Melanie Neumann wird im Modul „Beruf und Familie“ über neue Berufsmodelle referieren und Gelegenheit bieten, über wichtige Fragen und Sorgen in Hinblick auf eine Praxisgründung oder das Angestelltbleiben zu sprechen. Rechtsanwalt Michael Lennartz widmet sich beim Thema "Angestellt oder Chef" dem Übergang vom Studium in den Beruf und dann in die Freiberuflichkeit, erläutert Vorteile und Nachteile und wird sicherlich mit den Teilnehmern über das Thema „Flexibilität versus Sicherheit“ reden. Prozess- und Qualitätsmanager Sascha Kötter hat alles im Köcher, was die Teilnehmer zur finanziellen Sicherheit wissen müssen. Es geht nicht nur um die Frage, wie man nach einer Praxisgründung ruhig schlafen kann, sondern vor allem darum zu wissen, wofür man eigentlich morgens aufsteht. Rhetorik-Trainer Peter Edwin Brand wird in seinem Modul viel über das Thema „Erfolgreich kommunizieren“ vermitteln; im Mittelpunkt steht die Kommunikation mit Patienten, Chef und Mitarbeitern. Von den Teilnehmern selbst wird ebenfalls intensive Kommunikation gefragt sein: In Workshops arbeiten sie nach den jeweiligen Impulsvorträgen gemeinsam an den Themen weiter. Moderatoren werden die Teams bei der Erarbeitung ih- rer Themengebiete unterstützen. Zum Abschluss stellen die Gruppen ihre Ergebnisse im Plenum vor.

Das richtige Ambiente für das Zukunftsforum bietet an dem Wochenende das moderne Design-Tagungshotel „nhow Berlin“. Die Teilnahmegebühr beträgt für FVDZ-Mitglieder 149 Euro, für Nicht-Mitglieder 199 Euro. Studierende können für 25 Euro (Mitglieder) oder 50 Euro (Nicht-Mitglieder) an der Veranstaltung teilnehmen. Übernachtungskosten sind nicht in den Kongressgebühren enthalten. Anmeldungen sind im Internet unter www. fvdz.de/forum-zukunft.html möglich. Weitere Informationen zum Forum Zukunft oder zur Anreise gibt es bei Frauke Garstka in der FVDZ-Bundesgeschäftsstelle in Bonn unter der Telefonnummer 0228 / 855732 oder per Mail unter fg@fvdz.de. sas

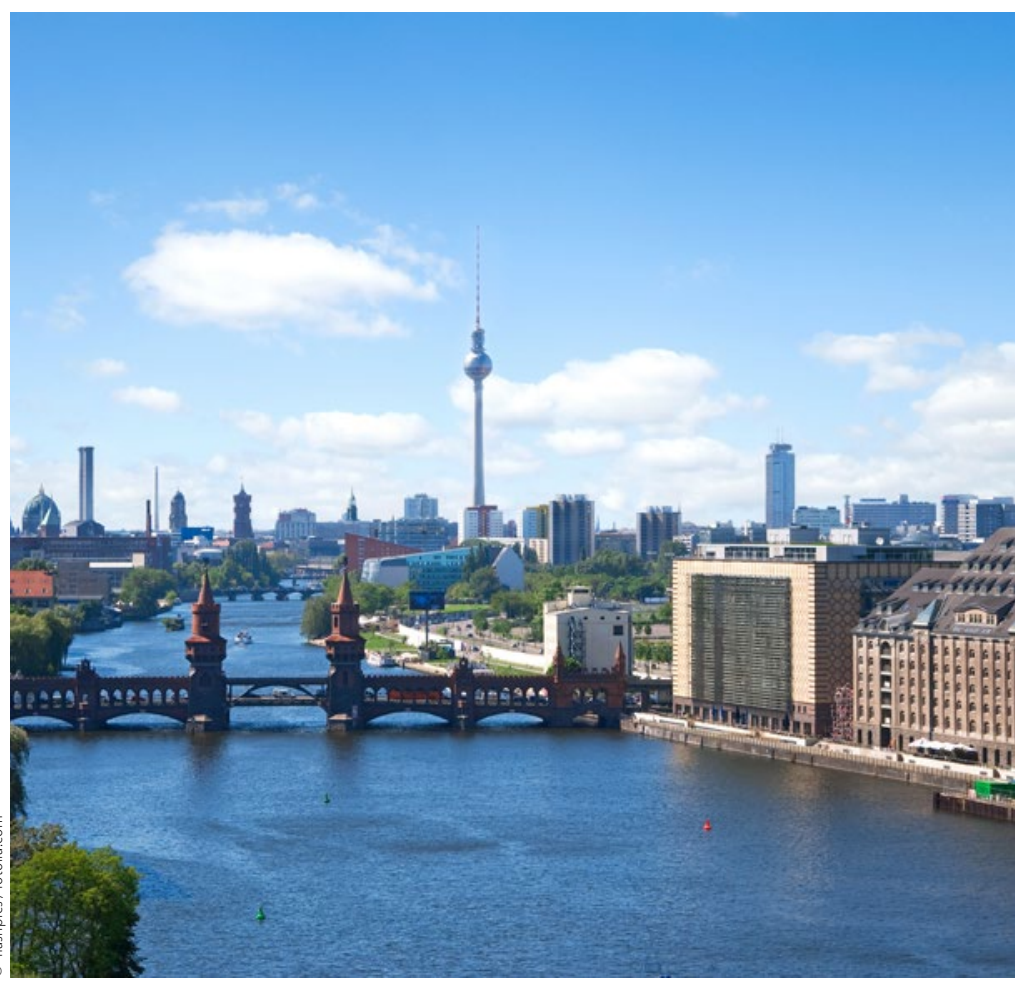

Freiverbändler wird Zahnärzte-Chef in Sachsen-Anhalt

\section{Hünecke ist neuer Präsident der Landeszahnärztekammer}

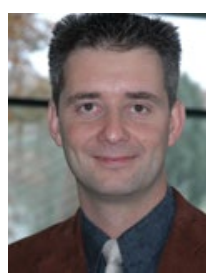

Die Delegierten der Landeszahnärztekammer Sachsen-Anhalt haben Dr. Carsten Hünecke aus Magdeburg zum Präsidenten der Landeszahnärztekammer gewählt. Der 52-Jährige wird Nachfolger von Frank Dreihaupt, der 26 Jahre lang an der Spitze der Landeszahnärztekammer Sachsen-Anhalt stand. Hünecke verfügt über

jahrzehntelange standespolitische Erfahrung. Er ist seit 23 Jahren Freiverbändler, war von 2001 bis 2011 Vorsitzender des FVDZ-Landesverbandes Sachsen-Anhalt und ist seither im Landesvorstand aktiv. In der Landeszahnärztekammer SachsenAnhalt gehörte Hünecke bereits als Referent für Öffentlichkeitsarbeit und Delegierter zur Bundesversammlung der Bundeszahnärztekammer dem bisherigen Kammervorstand an. cas 